\title{
The Study of Temperature and Humidity Data Logger Based on RX631
}

\author{
WeiWei Qu ${ }^{1, a}$, Hong Yang ${ }^{2, b}$, XiaoPing Zou ${ }^{3, c}$, HuiYong Liu ${ }^{4, d}$ \\ ${ }^{1,2,3,4}$ College of Science, Beijing Information Science and Technology University, Jianxiangqiao \\ Campus, Beijing 100101, China \\ âwojiaoquweiwei@163.com, byyanghong@yeah.net, 'xpzou2014@163.com, '996324296@qq.com
}

Keywords: monitor, temperature and humidity, RX631, GSM

\begin{abstract}
In order to ensure food security, a design scheme about temperature and humidity data logger for small refrigerator that could monitor temperature and humidity of the environment in real time was proposed. The recorder uses 32-bit RX631designed by Company RENESAS as the controller chip and the paper will show the hardware and software of the product in detail and draw a conclusion. The recorder had a lot of advantages such as a larger range about temperature measurement, getting the information of temperature, humidity and working state whenever possible, even sending relevant information to users in time by GSM.
\end{abstract}

\section{Introduction}

It is well known to us that temperature and humidity have a close relationship to people's life and work, and influence us on various fields of the society, such as warehouse management, manufacturing, scientific research, etc. Meanwhile, with the rapid development of industry, agriculture, technology and the national economy, more and more people pay attention to the control and detection of temperature and humidity in their life, which is really needed by the market and daily life[1]. In fact, temperature and humidity data logger has been widely used in many trades that need the technology of monitoring temperature and humidity, which is called the "necessary weapon" in cold-chain transportation. The temperature and humidity data logger in market has some disadvantages like little storage space, narrow temperature range, hard maintainability, etc. In order to meet market demand, we designed a temperature and humidity data logger that could measure the data of temperature and humidity in real-time, then uploaded the stored data to personal computer by a serial port or a USB port. It even could analyze and process data in host computer and judge whether there are mistakes in observation or transport process. The product we designed has solved some problems, including widening the measurement range of temperature [2], searching for temperature and humidity in real time, recording data [3], etc.

\section{Hardware architecture}

\subsection{General overview.}

The recorder uses RX631 made by the Company RENESAS as the control chip. Meanwhile, because of modular architecture, it takes the control chip as the core to control all the modules to work. Actually, the data logger takes 32-bit MCU (RX631) as the central processing unit and detects the information about temperature and humidity by the temperature sensor PT1000 and humidity sensor SHT21. Then it stores all the data in EEPROM and displays them by liquid crystal display (LCD). When the EEPROM space storing data is over 512 byte, the recorder will send the data to SD card and manage the data in SD card by the system FAT32. At the same time, the designers have set the alarm condition. Once triggering the alarm condition, the recorder will send alarm messages to the user. On the other hand, the users can check the information about data and parameter anytime and anywhere. The system block diagram is shown in Figure 1. 


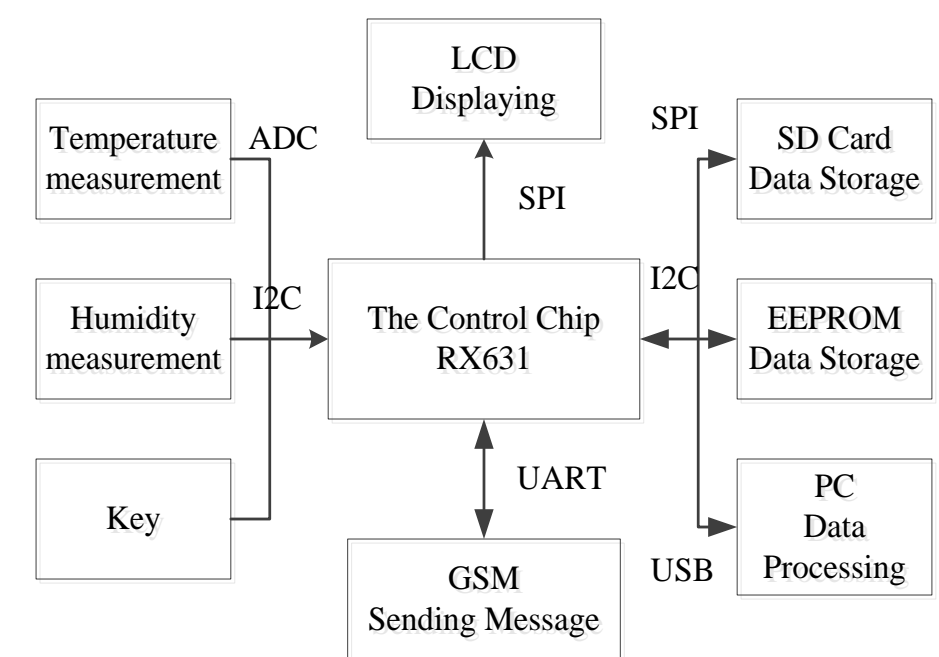

2.2 Temperature measurement

Fig. 1 The system block diagram

Figure 2 shows the circuit of temperature measurement. In the circuit, PT1000 is chosen as the temperature measurement resistance. In order to get the temperature measurement more accurate, the experimenter should make the voltage difference of resistance ends reach the highest point by changing the value of precision resistance RS[4].

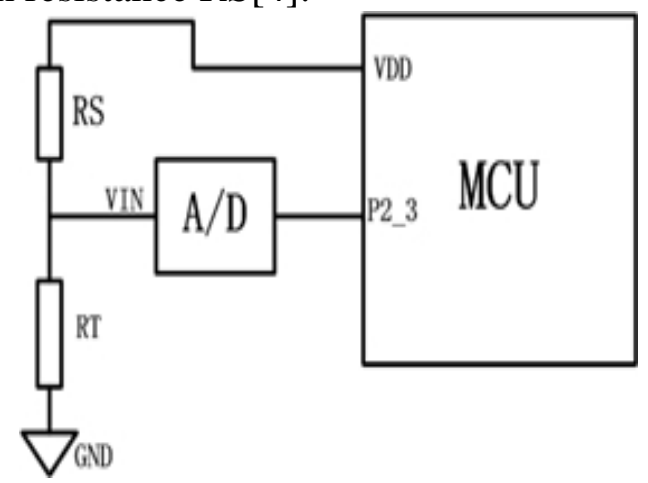

Fig.2 The circuit of temperature measurement

As is shown in Figure 2, RS, RT, VDD, GND and VIN are the precision resistance, temperature measurement resistance, the power supply voltage, ground and the value of resistance ends, respectively. Meanwhile, we assume that $\mathrm{RT}_{\max }$ is the maximum value of $\mathrm{RT}$ while $\mathrm{RT}_{\min }$ is the minimum value. After calculating, we know that $\Delta \mathrm{VIN}$ can peak and the temperature measurement will be the best accurate when the following equation works.

$$
\mathrm{RS}=\left(\mathrm{RT}_{\max } \times \mathrm{RT}_{\min }\right)^{1 / 2}
$$

2.3 Humidity measurement

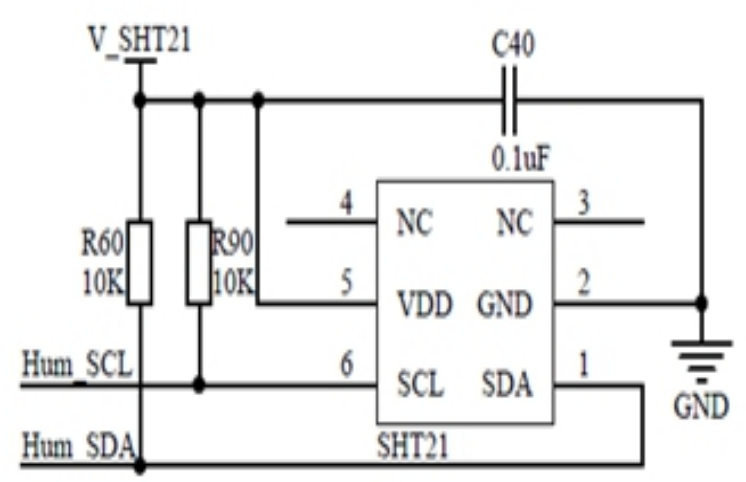

Fig. 3 The circuit of humidity measurement

The circuit of humidity measurement is shown in Figure 3. From it, we know that SHT21 made by Company SENSIRION is chosen as the humidity sensor. Through I2C communication mode, it takes use of the clock line SCL and SDA cable to send controlling information to the humidity 
sensor. What's more, the analog quantity of humidity is converted into the digital quantity by the internal AD in humidity sensor, so we can figure out the relative humidity according to the signal output by SDA cable and the following formula.

$\mathrm{RH} \%=\left(-6+125 \times \mathrm{S}_{\mathrm{RH}} / 216\right) \times 100 \%$

\subsection{Data storage}

In the experiment, we take 64KB AT24C512 produced by Company ATMEL as EEPROM Memory, which is called serial programmable memory that can be cleaned by electricity. The communication mode of EEPROM that stores the data of the system is I2C [5]. When mentioning SD card storage circuit, we pick 8GB SD card, whose communication mode is SPI and disk format is FAT32. Moreover, application file system can sort out all kinds of information, like temperature, humidity, log, address list and so on, which is benefit to read [6]. The circuit is given in Figure 4.

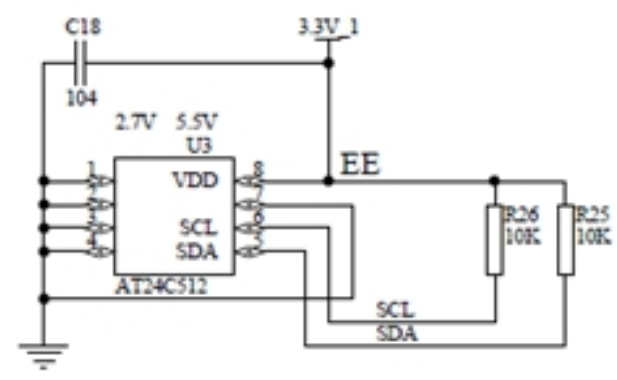

(a)

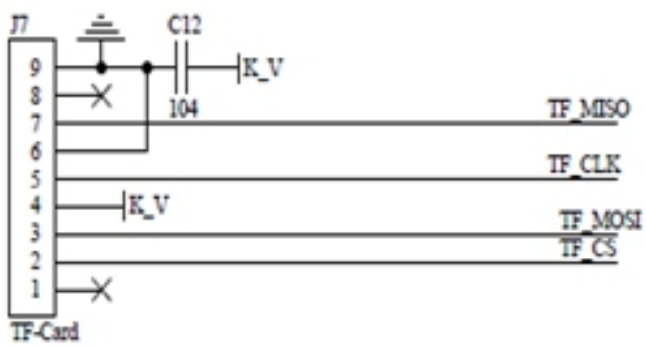

(b)

\subsection{GSM}

Fig. 4 The circuits of EEPROM data storage and SD card data storage

GSM module is the one that can connect GSM network, as well as making a call or sending message. In this product, we choose the smallest dual-band GSM / GPRS module (MC37iR3) made by Siemens in the market, which has 50 pins, including power pin, ground pin, communication pin connecting the control chip, SIM-connecting pin, LED-controlling pin and so on [7]. When GSM does not been connected to network successfully, LED will flash evenly (duty cycle is 50\%). On the contract, the lighting time accounts for $10 \%$ of the total time.

\section{Software architecture}

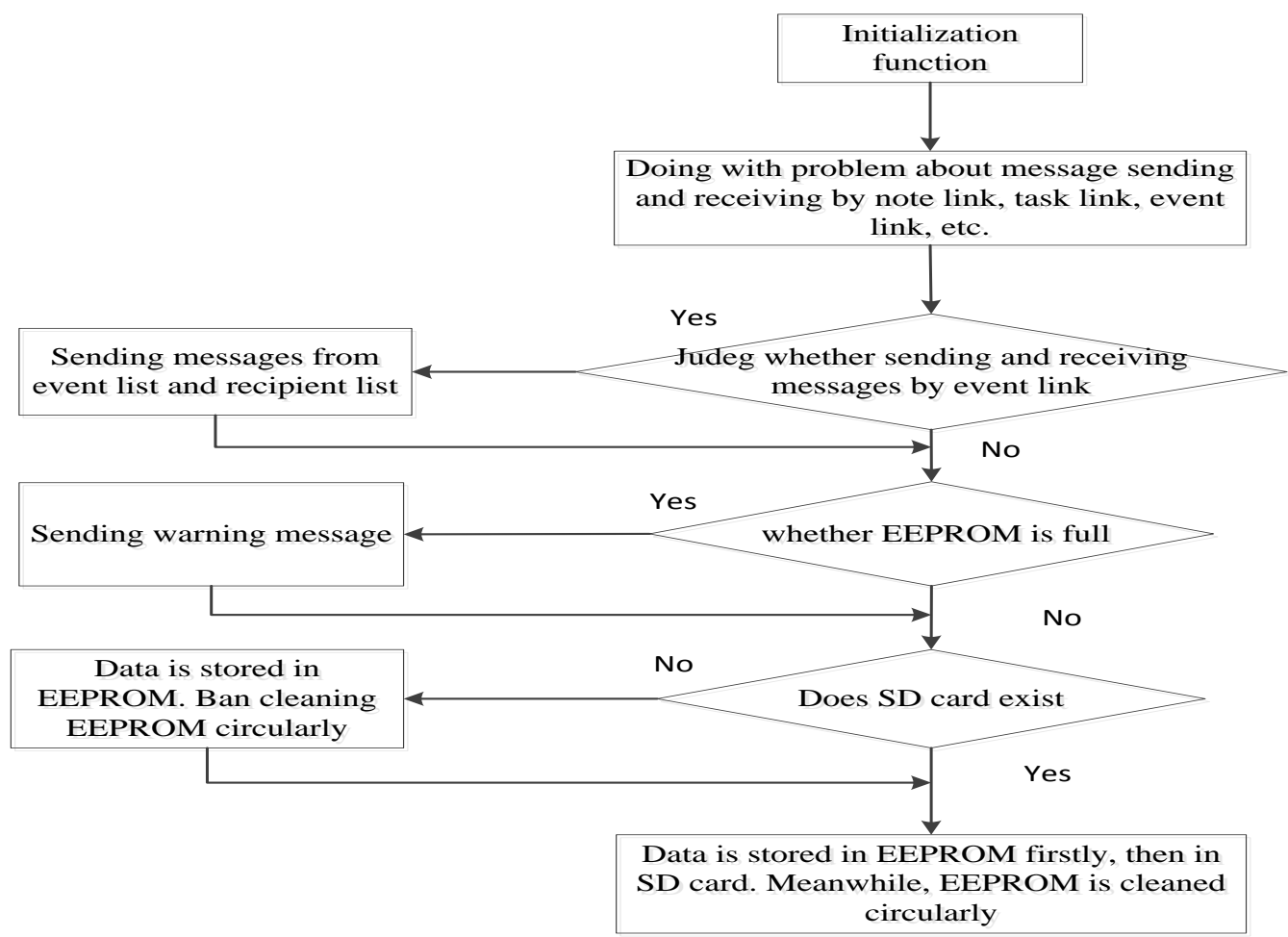

Fig. 6 the process diagram of software 
For the operator easy accessing to information, the design is required to set the sampling interval about temperature and humidity data, start time for recording, first warming time, alarm range and user's name and phone number by software in personal computer. Meanwhile, it can also output an Excel or cure graph from EEPROM where temperature and humidity data is stored and read storage data in SD card about temperature and humidity data, logs, contact lists, etc. Figure 6 is the process diagram of software. In the first stage, it consists of different initialization functions such as system, clock, interrupt, serial port, SD card, GSM module, etc. Then it enters into main cycle. In this part, it makes some judgments and performs actions about message, SD card, low power consumption, etc.

\section{Conclusion}

After testing the stability of the system, the experimenters are sure that all modular meet design requirements. There are several clear advantages in the temperature and humidity data logger.

(1) It uses platinum resistance as the temperature measurement sensor for widening the range of temperature measurement.

(2) It adds a function about GSM receiving-sending messages to help users to check temperature and humidity information as well as working information in real time.

(3) It increases a SD card to store more information and even makes use of FAT32 file system to manage the storage data, which are all for maintain the recorder.

\section{Conflict of Interests}

The authors declare that there is no conflict of interests regarding the publication of this paper.

\section{Acknowledgments}

This work was supported by BISTU Fund(5111523906).

\section{References}

[1] X. L. Yu , J. Yang , Y. Ni. Development of humidity sensor. Automotive technology and application, 2009, 02: 107 110.

[2] F. Xu. High-precision heating control device based on PT1000. Electrical application, 2013, 08: 20 23.

[3] P. Marwedel. Embedded system design - Embedded Systems Foundations of Cyber-Physical Systems, 2nd ed. Springer, 2011.

[4] B. L. Tian, C. Hu, F.L. Ding. A new method for improving the accuracy of PT1000 platinum resistance. Mechanical and electrical engineering, 2013, 05: 603 605.

[5] Z. J. Yang, Y. X. Wang. Interface technology of EEPROM and SCM based on I2C bus. Journal of Technology, 2001,02: 28 31.

[6] O. Elkeelany, V. S. Todakar. Data concentration and archival to SD card via hardware description language, 2011, 625 - 630.

[7] G. F. Gu, G. L. Peng. The survey of GSM wireless communication system. Computer and Information Application (ICCIA), 2010, 121 - 124. 